\title{
In Vacuo Measurement of the Sensitivity Limit of Planar Bragg Sensors
}

\author{
R. M. Parker ${ }^{1,2}$, J. C. Gates ${ }^{1}$, N. P. Sessions ${ }^{1}$, D. O. Kundys ${ }^{1}$, C. B. E. Gawith ${ }^{1}$, M. C. Grossel ${ }^{2}$, P. G. R. Smith ${ }^{1}$ \\ ${ }^{1}$ Optoelectronics Research Centre, ${ }^{2}$ School of Chemistry, University of Southampton, Highfield, Southampton, United Kingdom, SO17 1 BJ. \\ jcg@orc.soton.ac.uk
}

We present the direct measurement and modeling of the sensitivity limit of an integrated refractive index sensor for the detection of molecular monolayers. Direct UV writing can be used to fabricate a wide range of integrated optical devices particularly Bragg gratings. These Bragg gratings are inherently sensitive to temperature and strain. By etching a portion of the cladding, the mode within the grating region can be exposed to an analyte (Figure 1.a). The spectral response of the grating provides information about the refractive index of the analyte [1].

a)

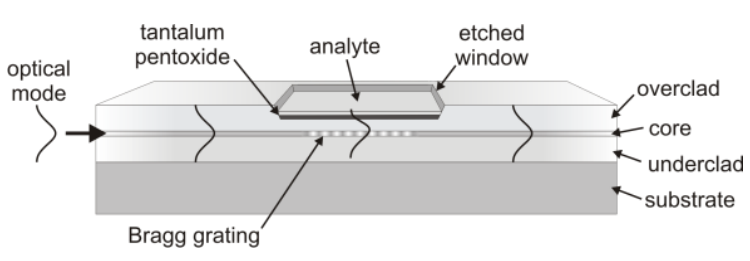

b)

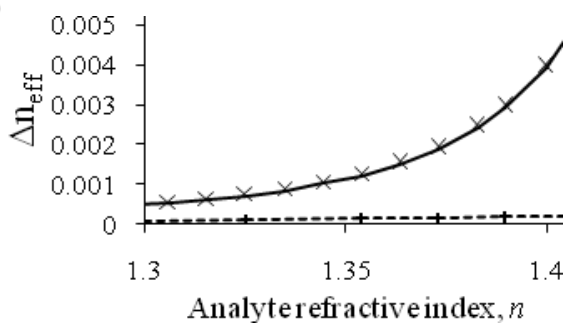

Fig. 1.a Schematic cross-section of the waveguide sensor. 1.b. The shift in effective refractive index for a Bragg grating when exposed to a series of analytes before (dashed) and after (solid) deposition of tantalum pentoxide (40 nm).

Adding a thin high index layer such as tantalum pentoxide increases sensitivity by pulling the mode into the analyte [2] (Figure 1.b). At these high sensitivities it becomes very difficult to make quantitative measurement of the absolute sensitivity limit in a lab environment. The challenge comes from minute surface adhesion effects and water absorption that dominate at the limits of detection.

a)

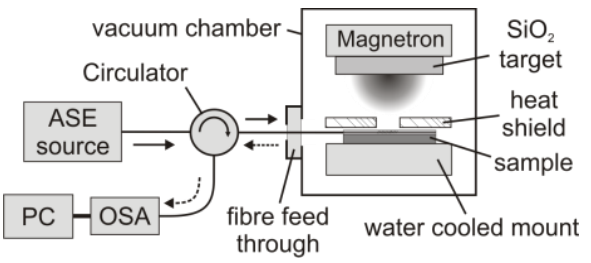

b)

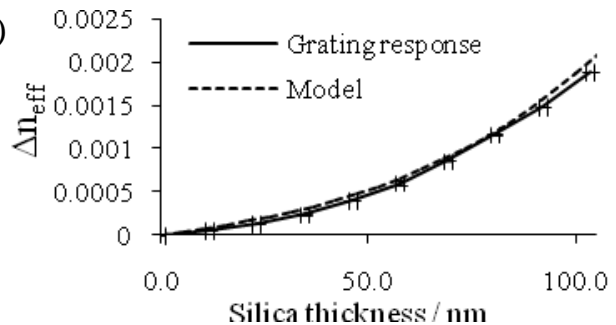

Fig. 2.a A schematic of the in vacuo measurement. 2.b The effective refractive index shift observed as a function of silica deposition onto the sensor surface.

Consequently to provide experimental verification of the sensitivity of the sensor, the response of the Bragg wavelength to the deposition of ultra-thin layers of silica in vacuo was investigated. The silica was sputtered using an Oxford Instruments Plasmalab 400 plus, allowing precise deposition of silica in 12nm steps (Figure 2.a). By measuring within the vacuum system it was possible to avoid surface contamination that would otherwise lead to further thickness changes (Figure 2.b). In order to compensate for thermal errors an additional local Bragg grating insensitive to the analyte is used.

Investigation of the experimental wavelength measurement uncertainty indicated a Bragg wavelength shift standard deviation of $1.18 \mathrm{pm}$ which corresponds to a silica thickness uncertainty of $0.25 \mathrm{~nm}$. This illustrates monolayer sensitivity, albeit in a vacuum environment. The index sensitivity is shown to be in the order of $10^{-6}$; an order of magnitude more sensitive than the shift expected to be observed on tethering a molecular monolayer onto the surface. Such a device will allow the study of surface wetting, functionalisation and ultimately monolayer binding within a microfluidic system.

\section{References}

[1] I.J.G. Sparrow, G. D. Emmerson, C.B.E. Gawith, P.G.R. Smith, M. Kaczmarek, A. Dyadyusha, "First order phase change detection using planar waveguide Bragg grating refractometer", Appl. Phys. B, 81, 1-4, (2005).

[2] G.R. Quigley, R.D. Harris, J.S. Wilkinson, "Sensitivity enhancement of integrated optical sensors by use of thin high-index films", Applied Optics, 38, 6036-6039, (1999). 